\title{
Utility of the pooling approach as applied to whole genome association scans with high- density Affymetrix microarrays
}

\author{
Alexandra Schosser ${ }^{1,4^{*}}$, Katrina Pirlo ${ }^{1}$, Darya Gaysina', Sarah Cohen-Woods ${ }^{1}$, Leonard C Schalkwyk ${ }^{1}$, Amanda Elkin ${ }^{1}$, \\ Ania Korszun², Cerisse Gunasinghe ${ }^{1}$, Joanna Gray ${ }^{1}$, Lisa Jones ${ }^{3}$, Emma Meaburn', Anne E Farmer ${ }^{1}$, Ian W Craig ${ }^{1}$, \\ Peter McGuffin ${ }^{1}$
}

\begin{abstract}
Background: We report an attempt to extend the previously successful approach of combining SNP (single nucleotide polymorphism) microarrays and DNA pooling (SNP-MaP) employing high-density microarrays. Whereas earlier studies employed a range of Affymetrix SNP microarrays comprising from $10 \mathrm{~K}$ to $500 \mathrm{~K} \mathrm{SNPs}$, this most recent investigation used the 6.0 chip which displays 906,600 SNP probes and 946,000 probes for the interrogation of CNVs (copy number variations). The genotyping assay using the Affymetrix SNP 6.0 array is highly demanding on sample quality due to the small feature size, low redundancy, and lack of mismatch probes.

Findings: In the first study published so far using this microarray on pooled DNA, we found that pooled cheek swab DNA could not accurately predict real allele frequencies of the samples that comprised the pools. In contrast, the allele frequency estimates using blood DNA pools were reasonable, although inferior compared to those obtained with previously employed Affymetrix microarrays. However, it might be possible to improve performance by developing improved analysis methods.

Conclusions: Despite the decreasing costs of genome-wide individual genotyping, the pooling approach may have applications in very large-scale case-control association studies. In such cases, our study suggests that highquality DNA preparations and lower density platforms should be preferred.
\end{abstract}

\section{Background}

We report an attempt to extend the previously successful approach of SNP (single nucleotide polymorphism) microarrays and DNA pooling (SNP-MaP) [1-14]. Whereas earlier studies had employed a range of Affymetrix SNP microarrays interrogating between $10 \mathrm{~K}$ to 500 K SNPs [15], we used the Affymetrix SNP 6.0 which displays 906,600 SNP probes and 946,000 probes for the interrogation of CNVs (copy number variations). We have performed this genome-wide association study (GWAS) using pooled DNA from a large depression case-control sample (1418 cases, 1301 controls), and the SNPs with the largest differences between cases and controls were individually genotyped in the sample used to

\footnotetext{
* Correspondence: alexandra.schosser@kcl.ac.uk

${ }^{1}$ MRC SGDP Centre, Institute of Psychiatry, King's College London, London, UK

Full list of author information is available at the end of the article
}

construct the pools. In contrast to the suitability of Affymetrix microarrays (up to the $500 \mathrm{~K}$ ) for successful analysis of DNA pools as established by other groups, the properties of the Affymetrix SNP 6.0 used in the current study were unproven at the time of investigation.

\section{Methods}

\section{Study design}

GWAS using phenotypically standardised pooled DNA of a large depression case-control (DeCC) sample was followed-up by individually genotyping the 'top-hit' SNPs. The SNP-MaP approach was conducted with the Affymetrix Genome-Wide Human SNP Array 6.0, containing approximately 1 million SNP markers [1].

\section{Samples}

Our depression case-control (DeCC) samples of 1418 patients with a diagnosis of recurrent major depression

\section{() Biomed Central}


(MDD) and 1301 control subjects were recruited from three clinical sites in UK (London, Cardiff and Birmingham) as described previously [16]. All participants gave written informed consent, and the study was approved by the Local Ethical Committees of the three centres.

\section{DNA pooling}

Blood samples were obtained from all patients and either blood or buccal mucosa swabs obtained from controls. Genomic DNA was extracted by an in-house validated procedure as described previously $[17,18]$. Genomic DNA was quantified three times using PicoGreen $^{\circ}$ assay (Molecular Probes, Eugene, OR, USA). The DNA pools of the DeCC sample were created as follows: first of all, the sample was divided into cases and controls, and then into males and females. Subsequently, these groups were divided according to their body mass index (BMI $<25$, BMI 25-30, BMI $>30)$ in the light of phenotypic analyses of DeCC, showing strong associations between depression and various physical diseases mediated via increased BMI [19]. We randomly created 57 DNA-pools of on average 47.67 individuals of matched sex and phenotype $(\mathrm{SD}=9.39)$, and each individual contributed $100 \mathrm{ng}$ DNA to their pools which ranged in concentrations from 19.0 to $24.99 \mathrm{ng} / \mu \mathrm{L}$.

\section{Allelotyping the DNA pools}

The Affymetrix Genome-Wide Human SNP Array 6.0 in combination with the standard Affymetrix protocol was used to allelotype $200 \mathrm{ng}$ genomic DNA from each pool. Water was used as a negative control to test for contamination, and to test for assay performance, the manufacturer's individual reference DNA was used as a positive control. Three pools were genotyped in duplicate, serving as technical replicates.

\section{Generating RAS (relative allele signal) scores}

In contrast to the earlier versions of Affymetrix genotyping arrays, the latest SNP arrays (Affymetrix Genome-Wide SNP Array 5.0 and 6.0; but not the previously employed $500 \mathrm{~K}$ chip) differ in that the mismatch probes have been discarded in favour of greater perfect match probe density. Thus, mismatch intensities can no longer be subtracted before calculating RAS scores, and RAS scores based on an equivalent principle to those that have been validated for pooling in previous work cannot, therefore, be calculated anymore. As a consequence they may no longer represent a good estimate of the absolute allele frequency in the pool; however, such estimates may still be useful for detecting frequency differences between pools [20].

\section{Confirmatory individual genotyping}

Confirmatory individual genotyping was performed using the Sequenom MassARRAY ${ }^{\bullet}$ iPLEX Gold assay and TaqMan ${ }^{\circ}$ SNP genotyping platform.

\section{Statistical analyses}

Cell intensity (.CEL) files were exported and RAS scores generated from the cell intensity data using the RAS score algorithm as implemented in a freely available script in R (http://sgdp.iop.kcl.ac.uk/oleo/affy), which has now become part of an $\mathrm{R}$ package, the SNPMaP package [21]. To test for significant differences between cases and controls, an independent Student's t-Test was applied using $\mathrm{R}$ statistical software (http://www.r-project.org/).

\section{Results}

We have performed a genome-wide association study (GWAS) using pooled DNA of a depression case-control sample and the Affymetrix Genome-Wide Human SNP Array 6.0, and followed-up the 'top-hit' SNPs using confirmatory individual genotyping with Sequenom MassARRAY ${ }^{\circ}$ iPLEX Gold or TaqMan ${ }^{\circ}$. Three DNA-pools were genotyped in duplicate and hybridized to two separate microarrays ('technical replicates'). The pairwise correlations were $0.945,0.965$ and 0.967 respectively. Biological replicates (pools of the same phenotypic group) were available for the majority of pools in the current study. We randomly selected five blood DNA case-pools of the same phenotypic group (cases, females, body-mass-index 25 to 30) and found an average pairwise correlation of 0.956; we found similar average pairwise correlations (0.959) for four cheek-swab-DNA control pools of the same phenotypic group (controls, females, body-mass-index less than 25) and four blood DNA control-pools of the same phenotypic group (0.967) (controls, females, body-mass-index less than 25). We allelotyped a total of 57 DNA pools of on average 47.67 individuals each ( $\mathrm{SD}=9.39), 29$ pools of cases and 28 pools of controls. All case-pools contained blood DNA, whereas only 10 control-pools contained blood DNA, the remainder (18) contained cheek swab DNA. To test for significant differences in allele frequencies between cases and controls, an independent Student's tTest was applied and after excluding rare variants with a minor allele frequency (MAF) $<0.05$, we found 74 SNPs crossing the genome-wide significance threshold of $7.2 \times 10^{-8}$ [22]. The top-ranked SNPs of the pooling GWAS were followed-up by individually genotyping the samples used to construct the pools, and the validity of the SNP-MaP approach was assessed by comparing allele frequency estimates from pooled DNA (RAS scores) with individual genotyping data of 110 SNPs 
genotyped with either Sequenom MassARRAY ${ }^{\bullet}$ iPLEX Gold (108 SNPs) or TaqMan ${ }^{\circ}$ (2 SNPs). We performed these analyses for cases and controls separately and, since all case pools contained blood DNA but 18 out of 28 control pools contained cheek swab DNA, we divided the controls into two groups according to source of DNA. The Pearson's correlation of the allele frequency estimates of the DNA pools (RAS scores) and the 'real' allele frequencies derived from individual genotyping was 0.9010 for the case-pools (all blood DNA) and 0.8853 for the blood DNA control-pools; however, the correlation was only 0.2734 for the cheek swab controlpools (see Figure 1).

\section{Conclusions}

The current study was an attempt to extend the previously successful approach of SNP microarrays and DNA pooling (SNP-MaP) to a whole-genome association study of MDD employing high-density microarrays. Whereas the earlier studies had employed a range of Affymetrix SNP microarrays comprising from $10 \mathrm{~K}$ to $500 \mathrm{~K}$ SNPs, this most recent investigation used the 6.0 chip, which displays 906,600 SNP probes and 946,000 probes for the interrogation of CNVs. The main advantage of DNA pooling is that it is a way to reduce costs in determining allele frequencies in large case/control cohorts. Since individual genotypes cannot be determined, this process is called "allelotyping" and it enables allele frequency differences between pools comprised DNA from many individuals to be compared without the need to genotype each individual.

For the purposes of allelotyping pooled DNA, there are three subtle but potentially detrimental changes differentiating the Affymetrix SNP 6.0 microarray from its validated $10 \mathrm{~K}, 100 \mathrm{~K}$ and $500 \mathrm{~K}$ predecessors. First, the feature size has decreased from $18 \mu \mathrm{m}$ on the $10 \mathrm{~K}$, to 8 $\mu \mathrm{m}$ on the $100 \mathrm{~K}$, and to $5 \mu \mathrm{m}$ on the $500 \mathrm{~K}$ and Affymetrix SNP 6.0 microarrays. Second, each SNP is interrogated by 40 probes on the Affymetrix $10 \mathrm{~K}$ and 100 $\mathrm{K}, 24$ probes for $90 \%$ of the Affymetrix $500 \mathrm{~K}$, but by only 6-8 probes on the Affymetrix SNP 6.0 microarray. Third, the mismatch probes have been discarded from the Affymetrix SNP 6.0 microarray, and thus mismatch probe intensities cannot be subtracted from perfect match probe intensities as it was the case for previous Affymetrix microarrays up to the $500 \mathrm{~K}$. To our knowledge, this is the first study published so far using the Affymetrix SNP 6.0 microarray on pooled DNA.

We created 57 DNA pools of the DeCC sample, each containing equal amounts of DNA of on average 47.67 individuals $(\mathrm{SD}=9.39)$.

We assessed validity of the genome-wide pooling study by comparing the estimates of allele frequencies from pooled DNA with individual genotyping data of 110 SNPs genotyped with either Sequenom MassARRAY $^{\circ}$ iPLEX Gold or TaqMan ${ }^{\circ}$, and allele frequency estimates provided by Affymetrix NetAff ${ }^{\mathrm{mm}}$ (http://www. affymetrix.com/analysis/index.affx). While there was a high correlation $(>0.99)$ between allele frequencies derived from confirmatory individual genotyping and the allele frequencies provided by Affymetrix NetAff ${ }^{\mathrm{mm}}$, the correlation between the allele frequency estimates derived from the pools (RAS scores) and the 'real' allele frequencies derived from individually genotyping the samples which composed the pools was somewhat disappointing. Although the correlation of RAS scores and 'real' allele frequencies was reasonable (but rather poor as compared to the previous Affymetrix microarrays up to the $500 \mathrm{~K}$ ) for the blood DNA cases (Pearson's correlation of 0.901) and the blood DNA controls (Pearson's correlation of 0.885 ), the correlation of cheek swab DNA controls was only 0.273 . The RAS scores produced using the Affymetrix SNP 6.0 array have a different distribution than the conventional RAS scores that had been validated for pooling in previous work, since the mismatch probes have been discarded in favour of greater perfect match probe intensity. Therefore, mismatch intensities cannot be substracted before calculating RAS scores, and the ratio of allele signal intensities $\mathrm{A} /(\mathrm{A}+\mathrm{B})$ is calculated instead. As a consequence, the RAS scores derived from the Affymetrix SNP 6.0 array are not necessarily a good estimate of the absolute allele frequency in the pool [21]. However, this should not affect case-control comparison in a genome-wide pooling study, since we are interested in frequency differences between the groups, not necessarily in absolute allele frequencies. Although the RAS scores indeed did not accurately predict the 'real' allele frequencies in our sample, we hypothesized that the difference between cases and controls are expected to be the same in the pools as in the individuals that comprise the pools. To test this hypothesis, we calculated the ratio of the mean allele frequencies of controls and cases (controls/cases) for the 110 SNPs individually genotyped, and the ratio of the mean RAS scores of control and case pools (control pools/case pools) for the same SNPs genotyped on the Affymetrix SNP 6.0 array. Subsequently, we calculated the ratio of the two ratios, where a ratio of 1 would indicate that the mean control/case ratio of the RAS scores for a given SNP was a perfect estimate of the 'real' ratio derived from individual genotyping of the samples composing the pools. We found that the mean of the latter ratios was 1.01 and the standard deviation (SD) was 0.25 , with $74.55 \%$ of SNPs within one SD. Performing the same analyses after separating blood and cheek swab controls, we found a mean of 0.99 and SD 


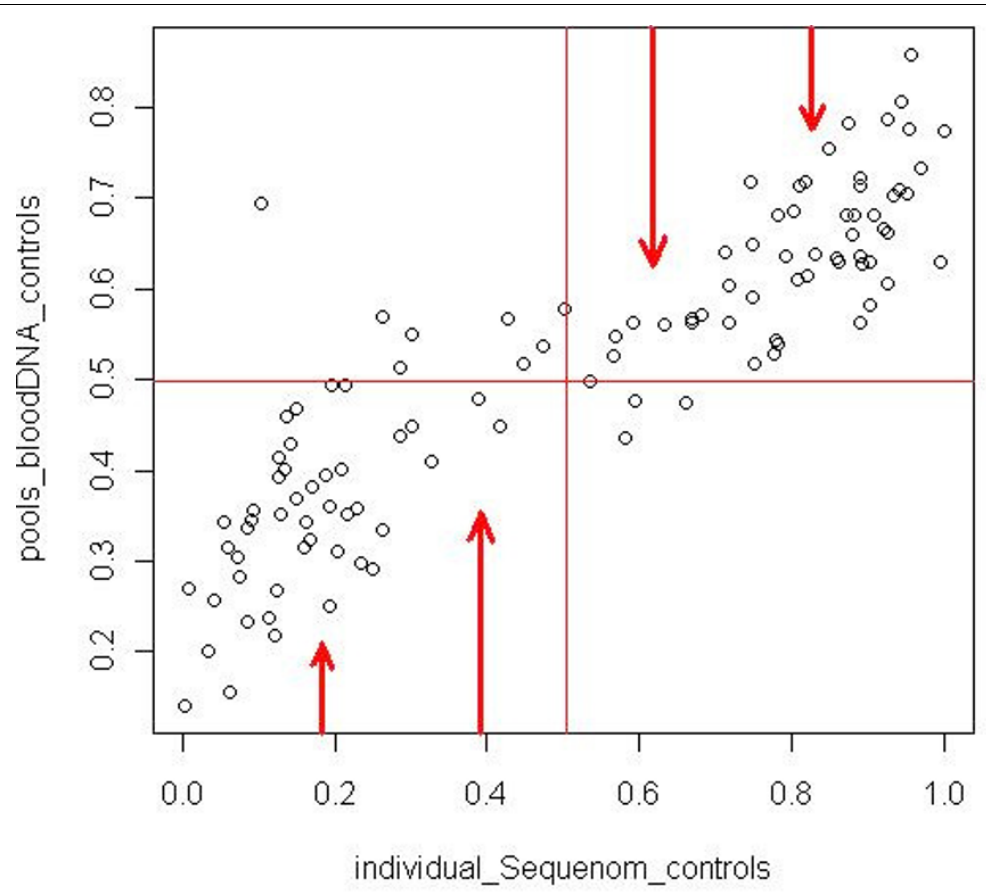

c)

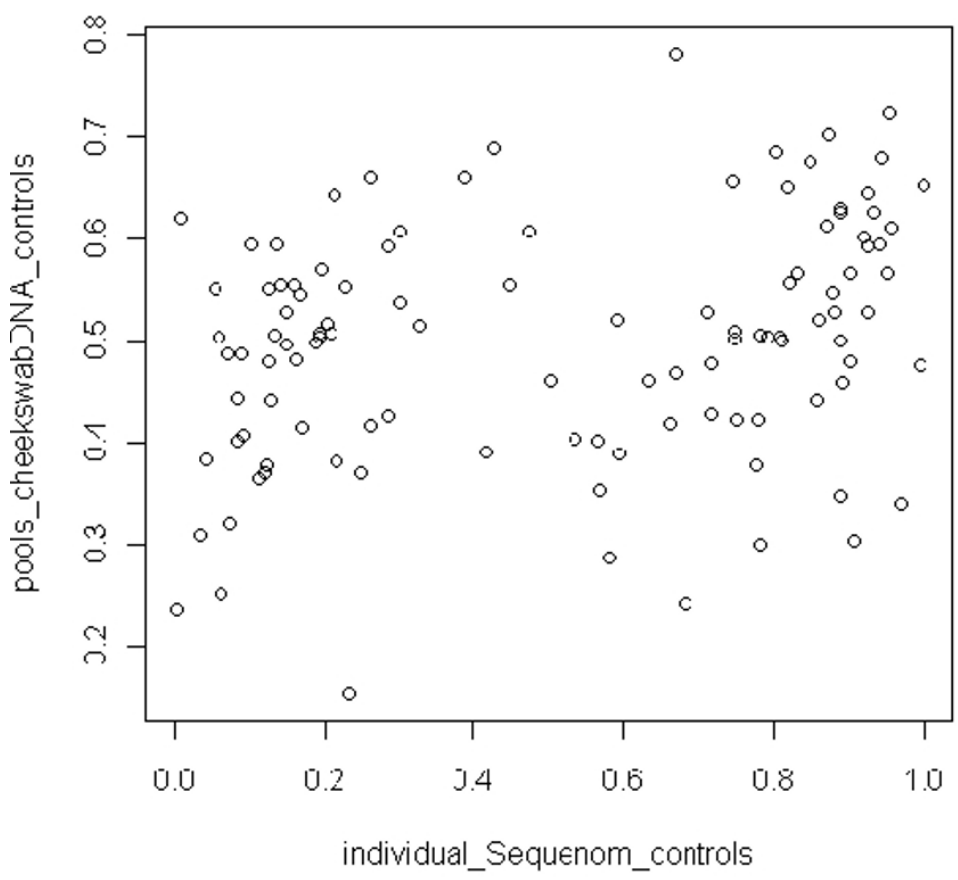

b)

Figure 1 Pearson's correlation of real allele frequencies (x-axis) and frequency estimates from pooled DNA (RAS-scores, $y$-axis) of BACCS controls (a) (blood DNA) and the DeCC controls (b) (cheek swab DNA) are shown. The red arrows (a) indicate, that the distribution of RAS scores (y-axis) are now slumped towards 0.5 as a consequence of the discarded mismatch probe (thus mismatch probe intensities cannot be substracted from perfect match probe intensities) as compared to previous Affymetrix microarrays up to the $500 \mathrm{~K}$. Pooled cheek swab DNA (b) could not sufficiently predict 'real' allele frequencies of the samples that comprised the pools. 
of 0.11 in case of blood controls (89.09\% of SNPs within $1 \mathrm{SD}$ ), and a mean of 1.05 and SD of 0.39 in case of cheek swab controls (74\% of SNPs within 1 SD).

These findings suggests that the microarray allelotyping cheek swab DNA pools did not sufficiently predict 'real' allele frequencies of the samples comprising the pools (see Figure 1) even though the quality of cheek swab DNA employed was comparable to that in previous studies. The genotyping assay using the Affymetrix SNP 6.0 array is highly demanding on sample quality due to the small feature size, low redundancy, and lack of mismatch probes. In contrast to the problems encountered with cheek swab DNA, the allele frequency estimates using blood DNA pools on the 6.0 chip were reasonable, although inferior compared to those obtained with previous Affymetrix microarrays up to the $500 \mathrm{~K}$ chip. Nevertheless, knowing the nature of the problems encountered and the differences in the organisation of the probe features distinguishing the Affymetrix SNP 6.0 array from its predecessors, it might be possible, however, to improve performance by developing improved analysis methods. In addition, despite the decreasing costs of genome-wide individual genotyping, the pooling approach with this (and other arrays) may still be applicable in very large-scale case-control association studies, preferably using high-quality blood DNA or a less SNP dense platform as appropriate.

\section{Acknowledgements}

Dr. Schosser was supported by an Erwin-Schrödinger-Fellowship (Ref. Nr. J2647) of the Austrian Science Funds and by a NIHR South London and Maudsley Biomedical Research Centre Preparatory Clinician Scientist Fellowship. Dr. Gaysina was supported by INTAS Postdoctoral Fellowship (Ref. Nr 04-83-3802) and Russian Science Support Foundation. The depression case control collection (DeCC) was funded by the MRC UK. Dr. Cohen-Woods was supported by a Medical Research Council (MRC) UK PhD studentship and a NIHR South London and Maudsley Biomedical Research Centre Postdoctoral Research Fellowship.

\section{Author details \\ ${ }^{1}$ MRC SGDP Centre, Institute of Psychiatry, King's College London, London, UK. ${ }^{2}$ Centre for Psychiatry, Wolfson Institute of Preventive Medicine, Barts and The London Medical School, Queen Mary University of London, London, UK. ${ }^{3}$ Department of Psychiatry, University of Birmingham, Birmingham, UK. ${ }^{4}$ Division of Biological Psychiatry, Department of Psychiatry and \\ Psychotherapy, Medical University Vienna, Vienna, Austria.}

\section{Authors' contributions}

AS carried out the molecular genetic studies, the statistical analyses and drafted the manuscript; KP performed pool construction and quantification; DG participated in quantification and genotyping. SCW participated in pool construction; LS supervised statistical analyses; AE, A, CG, JG and LJ participated in sample collection; EM supervised microarray genotyping; $A F$, IC and PM were principal investigators of the proposed study. All authors read and approved the final manuscript.

\section{Competing interests}

The authors declare that they have no competing interests.

\section{References}

1. Butcher LM, Meaburn E, Liu L, Fernandes C, Hill L, Al-Chalabi A, et al: Genotyping pooled DNA on microarrays: a systematic genome screen of thousands of SNPs in large samples to detect QTLs for complex traits. Behavior Genetics 2004, 34:549-555.

2. Butcher LM, Meaburn E, Knight J, Sham PC, Schalkwyk LC, Craig IW, et al: SNPs, microarrays and pooled DNA: identification of four loci associated with mild mental impairment in a sample of 6000 children. Hum Mol Genet 2005, 14:1315-1325.

3. Butcher LM, Davis OSP, Craig IW, Plomin R: Genome-wide quantitative trait locus association scan of general cognitive ability using pooled DNA and $500 \mathrm{~K}$ single nucleotide polymorphism microarrays. Genes Brain Behav 2008, 7:435-446.

4. Butcher LM, Plomin R: The Nature of Nurture: A Genomewide Association Scan for Family Chaos. Behav Genet 2008, 38:361-371.

5. Bansal A, van den Boom D, Kammerer S, Honisch C, Adam G, Cantor CR et al: Association testing by DNA pooling: an effective initial screen. Proc Natl Acad Sci USA 2002, 99(26):16871-16874.

6. Craig DW, Stephan DA: Applications of whole-genome high-density SNP genotyping. Expert Rev Mol Diagn 2005, 5:159-170.

7. Craig JE, Hewitt AW, McMellon AE, Henders AK, Ma L, Wallace $L$, et al: Rapid inexpensive genome-wide association using pooled whole blood. Genome Res 2009, 19(11):2075-2080.

8. Johnson C, Drgon T, Liu QR, Walther D, Edenberg H, Rice J, et al: Pooled association genome scanning for alcohol dependence using 104,268 SNPs: validation and use to identify alcoholism vulnerability loci in unrelated individuals from the collaborative study on the genetics of alcoholism. Am J Med Genet B Neuropsychiatr Genet 2006, 141B(8):844-853.

9. Liu QR, Drgon T, Johnson C, Walther D, Hess J, Uhl GR: Addiction molecular genetics: 639,401 SNP whole genome association identifies many "cell adhesion" genes. Am J Med Genet B Neuropsychiatr Genet 2006, 141B(8):918-925

10. Kirov G, Nikolov I, Georgieva L, Moskvina V, Owen MJ, O'Donovan MC: Pooled DNA genotyping on Affymetrix SNP genotyping arrays. BMC Genomics 2006, 15:7-27.

11. Yang HC, Liang YJ, Huang MC, Li LH, Lin CH, Wu JY, et al: A genome-wide study of preferential amplification/hybridization in microarray-based pooled DNA experiments. Nucleic Acids Res 2006, 34(15):e106.

12. Steer S, Abkevich V, Gutin A, Cordell HJ, Gendall KL, Merriman ME, et al: Genomic DNA pooling for whole-genome association scans in complex disease: empirical demonstration of efficacy in rheumatoid arthritis. Genes Immun 2007, 8(1):57-68.

13. Wilkening S, Chen B, Wirtenberger M, Burwinkel B, Försti A, Hemminski K, et al: Allelotyping of pooled DNA with $250 \mathrm{~K}$ SNP microarrays. BMC Genomics 2007, 8:77.

14. Shifman S, Bhomra A, Smiley S, Wray NR, James MR, Martin NG, et al: A whole genome association study of neuroticism using DNA pooling. Mol Psychiatry 2008, 13:302-312.

15. Meaburn E, Butcher LM, Liu L, Fernandes C, Hansen V, Al-Chalabi A, et al: Genotyping DNA pools on microarrays: tackling the QTL problem of large samples and large numbers of SNPs. BMC Genomics 2005, 6(1):52.

16. Schosser A, Gaysina D, Cohen-Woods S, Chow PC, Martucci L, Craddock N, et al: Association of DISC1 and TSNAX genes and affective disorders in the depression case-control (DeCC) and bipolar affective case-control (BACCS) studies. Mol Psychiatry 2009.

17. Freeman B, Powell J, Ball D, Hill L, Craig I, Plomin R: DNA by mail: an inexpensive and noninvasive method for collecting DNA samples from widely dispersed populations. Behav Genet 1997, 27:251-257.

18. Freeman B, Smith N, Curtis C, Huckett L, Mill J, Craig IW: DNA from buccal swabs recruited by mail: evaluation of storage effects on long-term stability and suitability for multiplex polymerase chain reaction genotyping. Behav Genet 2003, 33(1):67-72.

19. Farmer A, Korszun A, Owen MJ, Craddock, Jones L, Jones I, et al: Medical disorders in people with recurrent depression. Br J Psychiatr 2008, 192:351-355.

20. Pearson JV, Huentelman MJ, Halperin RF, Tembe WD, Melquist S, Homer N, et al: Identification of the genetic basis for complex disorders by use of pooling-based genomewide single-nucleotide polymorphism association studies. Am J Hum Genet 2007, 80:126-139. 
21. Davis OS, Plomin R, Schalkwyk LC: The SNPMaP package for R: a framework for genome-wide association using DNA pooling on microarrays. Bioinformatics 2009, 25:281-283.

22. Dudbridge F, Gusnanto A: Estimation of significance thresholds for genomewide association scans. Genet Epidem 2008, 32:227-234.

doi:10.1186/1756-0500-3-274

Cite this article as: Schosser et al: Utility of the pooling approach as applied to whole genome association scans with high-density Affymetrix microarrays. BMC Research Notes 2010 3:274.

Submit your next manuscript to BioMed Central and take full advantage of:

- Convenient online submission

- Thorough peer review

- No space constraints or color figure charges

- Immediate publication on acceptance

- Inclusion in PubMed, CAS, Scopus and Google Scholar

- Research which is freely available for redistribution

Submit your manuscript at www.biomedcentral.com/submit
C Biomed Central 